\title{
Ground calibration of MAPMT and SiPM for JEM-EUSO
}

\author{
Michael Karus*, Francesca Bisconti, Thomas Huber, Andreas Ebersoldt, Andreas \\ Haungs, Harald Schieler, Andreas Weindl
}

Karlsruhe Institute of Technology (KIT) - Institute for Nuclear Physics (IKP)

E-mail: michael.karusakit.edu

\section{for the JEM-EUSO Collaboration}

In order to unveil the mystery of ultra-high energy cosmic rays (UHECRs), JEM-EUSO (Extreme Universe Space Observatory on-board Japanese Experiment Module) will observe extensive air showers induced by UHECRs from the International Space Station (ISS) orbit with a huge acceptance. To discuss the origin of UHECRs precisely with the observed results, it is essential to calibrate the detector pre-flight with utmost precision.

The technical readiness level of JEM-EUSO is demonstrated with several pathfinder missions, e.g. EUSO-Balloon, EUSO-TA, and Mini-EUSO. These pathfinder missions also provide the perfect environment to investigate the capability of new state of the art photo sensors based on Geiger mode avalanche photodiodes (G-APDs), so called silicon photomultipliers (SiPMs). A first concept design for a JEM-EUSO elementary cell based on SiPMs is presented to be used with one of the pathfinder experiments. For the case of EUSO-TA comparison studies have been performed for MAPMT- and SiPM-response to fluorescence light.

To absolutely calibrate and characterize different types of photo sensors, an universal calibration and test setup is built at the Karlsruhe Institute of Technology (KIT). It consists of a photon shielding dark box $(1.5 \mathrm{~m} \times 1 \mathrm{~m} \times 1 \mathrm{~m})$, a diffuse uniform light source with a calibrated optical output and several readout electronics for MAPMTs and SiPMs. The light source can be used in singlephoton mode (pulsed light) and other modes, ranging from a few tens of photons to continuous light. The spectral output is around $(377 \pm 6) \mathrm{nm}$. Further wavelengths will be implemented with respect to the emission lines of the nitrogen fluorescence spectrum.

Former measurements with SiPMs have shown a temperature dependence of dark count and gain. Therefore, a temperature control has been implemented into the setup. With this the photo detection efficiency of various photo sensors can be measured for different wavelengths and temperatures. The present status of the calibration and test setup as well as of the design and comparison studies with SiPMs will be reported.

The 34th International Cosmic Ray Conference,

30 July- 6 August, 2015

The Hague, The Netherlands

\footnotetext{
* Speaker.
} 


\section{Introduction}

The JEM-EUSO mission aims for the space-based UV-fluorescence detection of ultra-high energy cosmic rays (UHECRs), using Earth's atmosphere as a calorimeter, measuring fluorescence light and Cherenkov light emitted by UHECR-induced extensive air showers (EASs). The main goal of the mission is to deepen the understanding of the cosmic ray flux spectrum above energies of $5 \times 10^{19} \mathrm{eV}$, where the extragalactic component is supposed to be dominant. Further scientific goals are the identification of sources and source regions for UHECRs. JEM-EUSO will make use of the multi-messenger approach examining neutrinos, $\gamma$-rays, the galactic magnetic fields, meteors, meteoroids, and Earth's atmosphere [1,2].

One of the pathfinder experiments for JEM-EUSO, namely EUSO-TA, provides a suitable environment to investigate the capability of silicon photomultipliers (SiPMs). EUSO-TA consists of one photo-detector module (PDM $=6 \times 6$ MAPMTs divided in 9 elementary cells) and two Fresnel lenses [3]. It is placed in front of one of the fluorescence detectors of the Telescope Array (Utah, USA). With its field-of-view of $11^{\circ}$, it is facing an electron light source and a LIDAR, for relative on-site calibration. To test SiPMs there, a first concept of an elementary cell (EC) based on SiPMs was designed (Section 2). Further, comparison studies were performed to evaluate the response of current MAPMTs and SiPMs to fluorescence light (Section 4).

\section{Concept of a SiPM-based elementary cell}

The new SiPMs have, compared to the classic PMTs, similar PDE and additional advantages like a better time resolution, a more compact structure, and a low operation voltage [4]. These features make SiPMs a possible photodetector for new space-based astroparticle observatories.

A promising candidate for a SiPM-based focal surface is the new 64-pixel TSV-MPPC (through silicon via multi-pixel photon counter) array series from Hamamatsu [5]. The feature of this new kind of SiPMs is that the anode and cathode are wired through the substrate. This technique allows to reduce the dead space between two pixels from $3 \mathrm{~mm}$ to $0.2 \mathrm{~mm}$ so that there is no more need to mount Winston cones to increase the active area.

A CAD design of a SiPM-based EC prototype was made and 3D-printed (Figure 1). The TSV-MPPC candidate fits into the former design of the MAPMT-based EC. With this it is possible to create a plane PDM focal surface with MAPMT-ECs and TSV-MPPC-ECs side by side. This will be used to compare the performance of both, in experiments like Mini-EUSO, EUSO-TA and EUSO-Balloon. Because of the reduced size and lower mass of TSV-MPPCs compared to MAPMTs, the SiPM-EC could host an additional board for temperature measurement or amplifiers.

\subsection{Temperature dependent measurements}

The behavior of the TSV-MPPCs at low temperatures is not well known. For a future use of these devices in space, this has to be studied. Several efforts are made at KIT to investigate the performance of TSV-MPPCs at different temperatures. For data acquisition of the temperature measurement, we use a Raspberry $\mathrm{Pi} \mathrm{B}+$ and a combined temperature and humidity sensor (AM2303), with communication via the $\mathrm{I}^{2} \mathrm{C}$ protocol. Several sensors are attached to a testing 


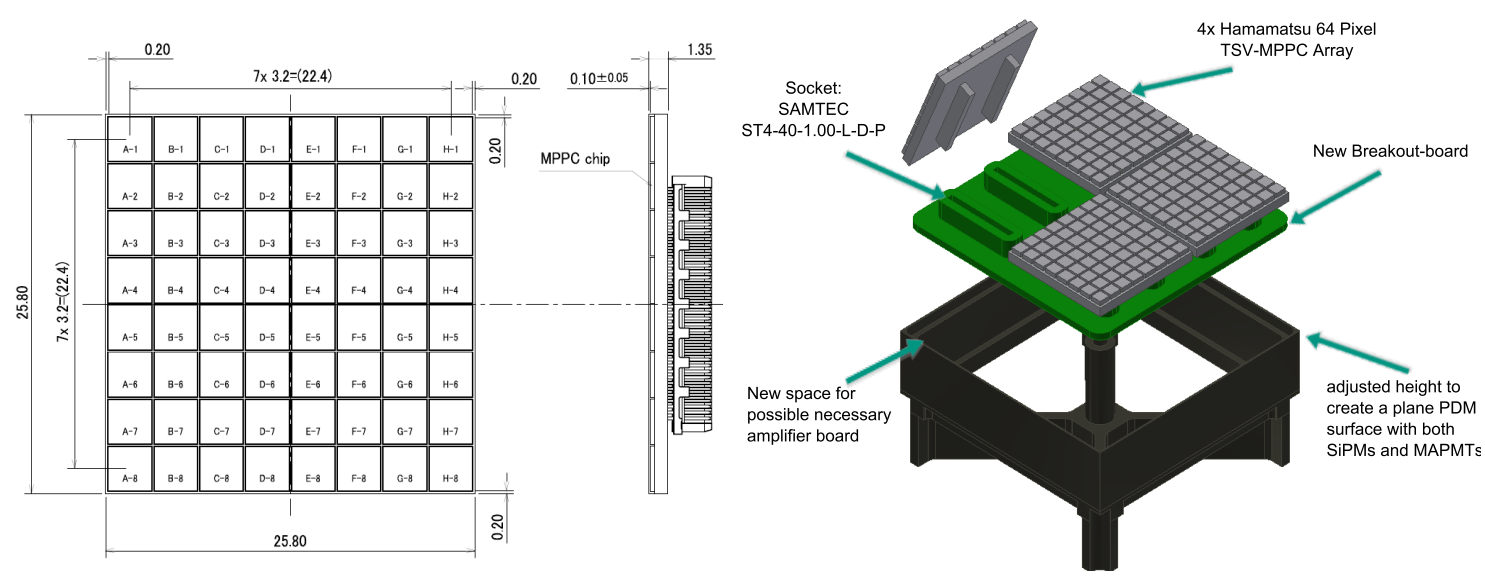

Figure 1: Left: Layout of the 64 pixel TSV-MPPC array S12642-0808PB-50. The geometrical dimension of four arrays fits with the former MAPMT-EC design [5]. Right: First prototype of a SiPM-based EC.

device for single-pixel TSV-MPPCs and can measure the temperature with an accuracy of $\pm 0.3{ }^{\circ} \mathrm{C}$ and humidity with $\pm 2 \%$ respectively, both with a sampling period of two seconds.

To cool down the TSV-MPPCs, a cold chamber and a modified freezer with a special no-frost technique is being prepared (Figure 2 left). The cold chamber is able to cool down to $-60^{\circ} \mathrm{C}$ and to program different temperature schedules. For these measurements we use a 3D-printed testing device that is coated with black flock paper as shown in Figure 2 right. The device is able to read out all the Hamamatsu TSV-series versions $\left(2 \times 2 \mathrm{~mm}^{2}, 3 \times 3 \mathrm{~mm}^{2}\right.$, and $\left.6 \times 6 \mathrm{~mm}^{2}\right)$. Investigating the $3 \times 3 \mathrm{~mm}^{2}$ TSV-MPPCs is of special interest, as this type is equal to one pixel from the 64 pixel array, our candidate for the SiPM-based EC. We started measuring the dark current and the dark noise of TSV-MPPCs in the cold chamber to compare the results with the characteristics of the old ceramic packed MPPCs. Their performance is suitable for our needs and was well examined in the past. However, their packaging does not allow a focal surface without gaps.

Since the cold chamber is not light-tight, it is not a suitable environment for absolute measurements. Therefore we are converting a small freezer to put it into the calibration stand and run the measurements mentioned in Section 3 at different low temperatures.

\subsection{SiPM readout}

Currently we are using a modified version of the Hamamatsu C12332 evaluation board for readout and connect it to a DRS4 evaluation board ${ }^{1}$. For data acquisition we are working on a command-line program. The program is ready to receive and process all four channels of the DRS4 board. Further features are: setting trigger-levels, having a free-running mode, changing the sampling rate and shifting the voltage range. Data can be saved in compressed .csv-files and in ROOT format.

Our next step is to integrate the modification of the bias voltage in order to stabilize TSVMPPCs with respect to small temperature fluctuations. To distinguish signal from noise when using

\footnotetext{
${ }^{1}$ http://www.psi.ch/drs/evaluation-board - June 2015
} 

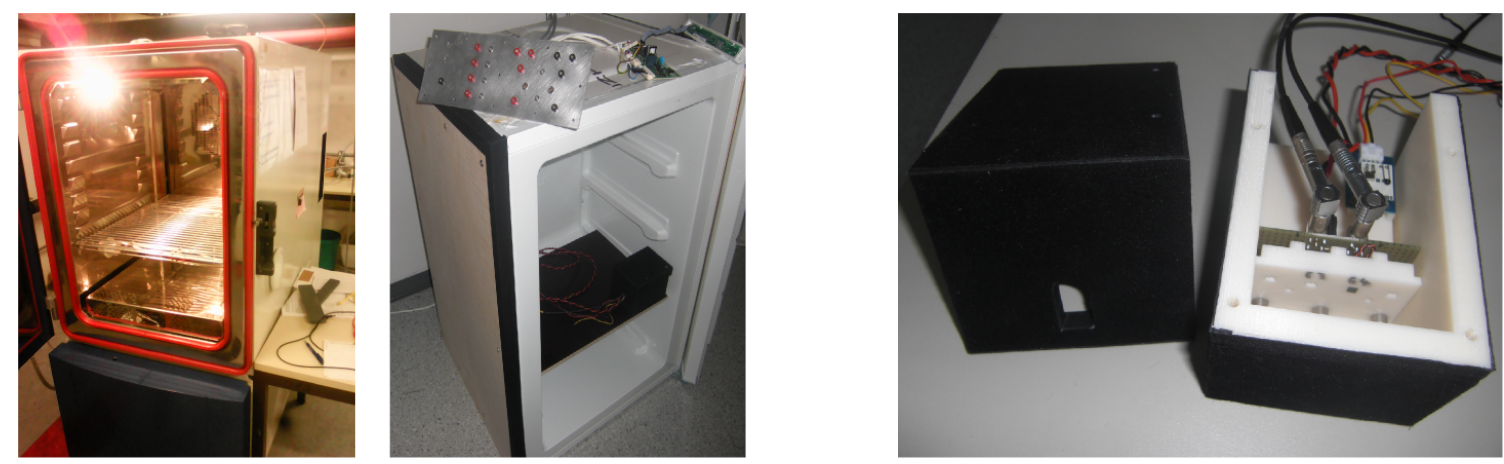

Figure 2: Left: Cold chamber from KIT-IPE for non-lightcalibrated measurements and modified freezer under construction. Right: Testing device to analyze single-pixel TSV-MPPCs of different sizes includes temperature and humidity sensor and one part of the modified Hamamatsu evaluation board. The photon shielding is surrounding the TSV-MPPC socket, overlapping the corners, to be light-tight.

SiPMs, there is also work ongoing to create a python-based peak-finding algorithm specialized for the peculiarities regarding crosstalk and afterpulses of SiPMs.

A prototype of a readout board for single pixels is in production. It includes an adjustable bias voltage, ports for three different types of single-pixel TSV-MPPCs, an amplifier, an integrated readout for energy measurements, and a fast readout to study the time resolution. As power source the MPPC power supply module C11204-01 is used. This module can output up to $90 \mathrm{~V}$ and contains the possibility to regulate the bias voltage on-the-fly. This is used to compensate for temperature fluctuations with a resolution of $1.8 \mathrm{mV}$ steps via a serial interface.

\section{Calibration}

To reliably estimate the energy of the primary particle from the EAS's UV-light signal, the detectors have to be calibrated in an absolute way in single-photon mode, with the highest precision possible. This is even more important for the space-based observation of EAS, where access to the detector is not given during the mission time. In the case of JEM-EUSO the focal surface will consist of MAPMTs or SiPMs, which have to be sorted pre-flight, according to their photo detection efficiency (PDE).

\subsection{Calibration stand}

For the calibration and sorting of the detectors, a calibration stand for single-photon generation and detection was built at the Institute for Nuclear Physics (IKP) at Karlsruhe Institute of Technology (KIT). It is based on a calibration stand built at APC (Paris) [6, 7]. It consists of a reference light source, the detector, photon shielding and readout electronics.

A schematic view of the set-up can be seen in Figure 3 left. Inside the photon shielding the reference light source is located. The UV-light $(\approx 377 \mathrm{~nm})$ from the LED-array is diffusely reflected inside the integrating sphere. The sphere's inside is made of PTFE (Polytetrafluoroethylene) which reflects $98 \%$ of UV-light in the region of $300-430 \mathrm{~nm}$.

The integrating sphere works as a beam splitter and a diffuser. Both exit-ports emit the same number of photons $\mathrm{N}_{\text {Sphere }}$. This is measured at one exit-port with a NIST-calibrated photodiode 

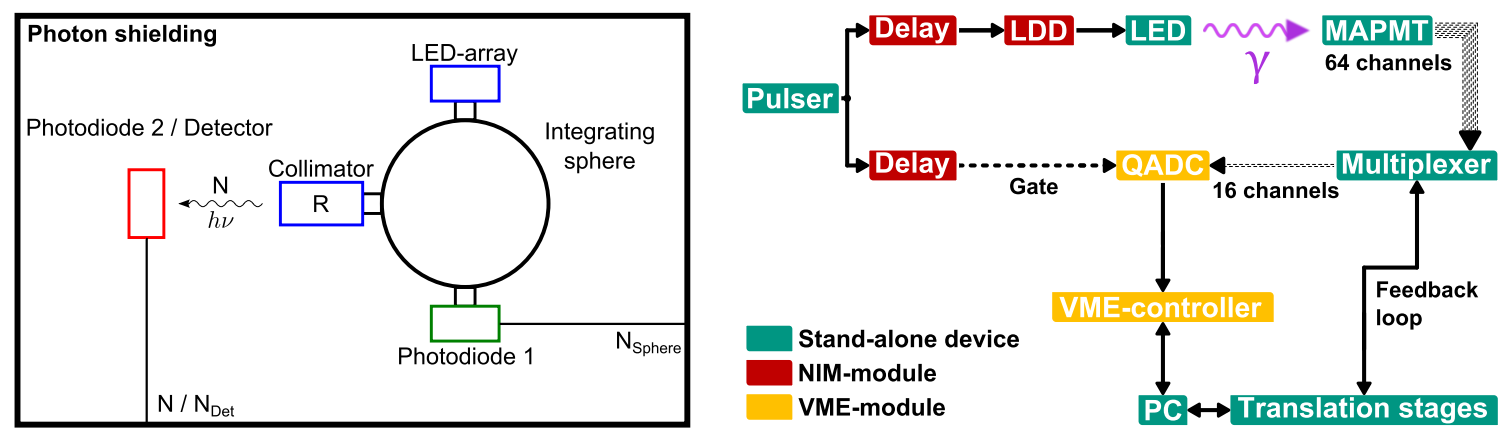

Figure 3: Schematic view of the calibration process. Left: The experimental set-up of the photon shielding $\left(2 \times 1 \times 0.9 \mathrm{~m}^{3}\right)$, the light source and the examined detector is shown. All ports on the integrating sphere are perpendicular to each other in the real set-up. Right: Schematic view of the signal chain of the on-ground calibration stand at KIT for MAPMTs.

(Photodiode 1). The collimator at the second exit-port reduces the photon flux from the exit-port. This is necessary because the light source will illuminate photo sensors with a gain around $10^{6}$ bigger than the gain of the photodiode.

The optical output of the light source is measured by a second NIST-calibrated photodiode (Photodiode 2) as the number of photons $\mathrm{N}$ that are emitted by the light source. The ratio of both photon numbers gives the collimator factor $\mathrm{R}$ of about $10^{-6}$. Because of the low gain of the photodiode and the strong collimator reduction, the whole LED-array is set to continuously emit light. The collimator factor R only depends on the collimator geometry and is measured very precisely with both photodiodes. The number of photons $\mathrm{N}$ can be calculated via the measurements of the number of photons inside the sphere. As a result the number of emitted photons $\mathrm{N}$ is known.

\subsection{Calibration process}

For high-gain detectors in front of the reference light source, only one LED of the LED-array is used while being pulsed by an LED-driver. A very low number of photons is emitted by the LED per pulse. The light source itself is aligned with the photo sensor and every single pixel (MAPMT: $2.88 \times 2.88 \mathrm{~mm}^{2}$; MPPC: $3 \times 3 \mathrm{~mm}^{2}$ ) will be scanned one by one. The exit hole of the collimator has a diameter of $0.5 \mathrm{~mm}$. With a distance of roughly under $2 \mathrm{~mm}$ we are sure that all photons that exit the collimator actually hit the sensitive detector area.

MAPMTs: The signal chain for MAPMTs is shown in Figure 3 right. The pulsing signal is duplicated and used to generate a gate signal for the data acquisition system. With the right timing via some delays, the charge-integrating analog-digital-converter (QADC) is only sensitive to inputs from an MAPMT when the gate signal is given, i. e. measurements are only taken when the MAPMT is illuminated by a light pulse. The QADC is controlled by a PC via a VME-controller which reads out the data. The computer also controls the multiplexer and the translation stages for positioning of the light source. The number of detected photons by an MAPMT pixel is N $\mathrm{N}_{\text {PMT }}$. The ratio of $\mathrm{N}_{\mathrm{PMT}}$ with the total number of photons sent to this pixel gives the photon detection efficiency of the MAPMT pixel. It is a product of the probability of the photo-cathode to emit an electron and the probability of that electron to reach the first dynode and to be multiplied. This 
whole process is described by Poisson statistics. This can be seen when recording a single photoelectron spectrum for single pixels. A schematic histogram of the measured QADC values is shown in Figure 4 left. Around the zero photoelectron peak ( 0 pe peak) there are dark counts of the MAPMT. This value is called the pedestal. At higher QADC values a one photoelectron peak (1 pe peak) is present. The values there correspond to one measured photoelectron. The resulting spectrum is a superposition of 0 pe, 1 pe, 2 pe, ... peaks.
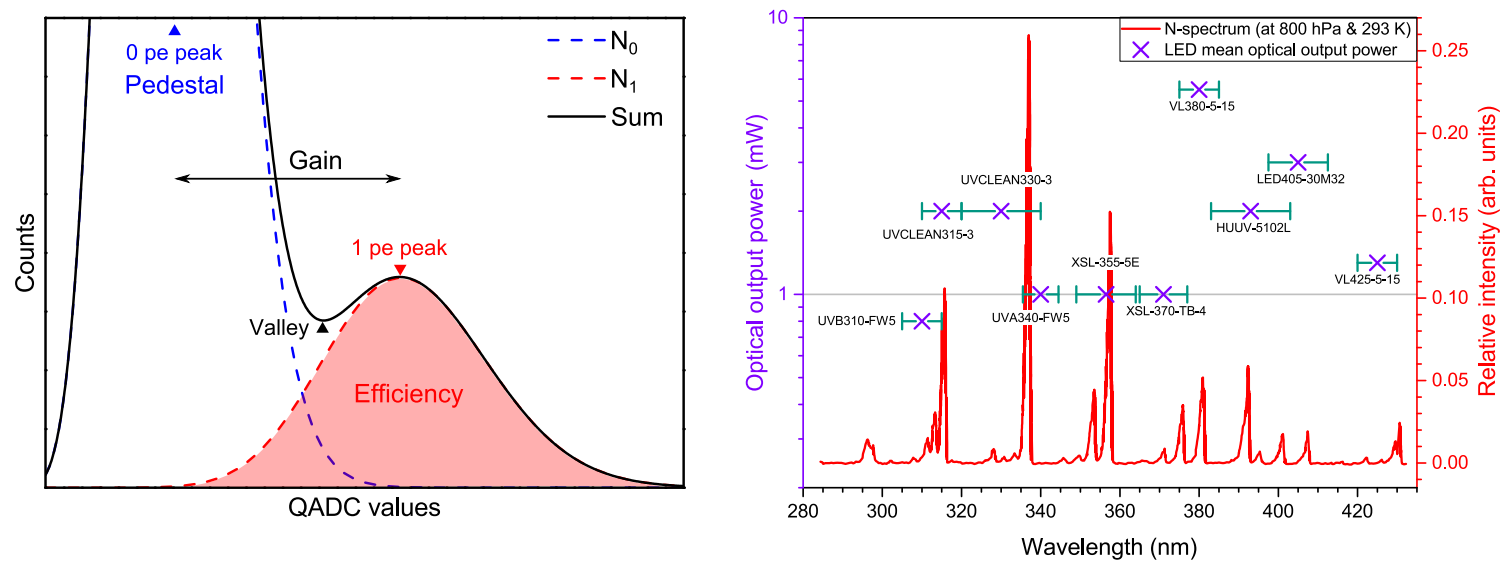

Figure 4: Left: Schematic sketch of a single photoelectron spectrum with two Poisson-distributions for the zero and one photoelectron peak. The separation of both peaks gives the gain of the detector. The area under the 1 pe peak gives the efficiency of the detector (adopted from [7]). Right: Fluorescence spectrum of nitrogen at $800 \mathrm{hPa}$ and $293 \mathrm{~K}$ with an overlay of UV-LED optical outputs (N-spectrum adopted from [8]).

The ratio of the total number of 0 pe counts to 1 pe counts should be higher than 50 . This assures that less than $1 \%$ of counts from a 2 pe peak are wrongly counted as 1 pe counts. That is an acceptable level for calibration. The gain can be calculated via the separation of the 0 pe and 1 pe peaks. The efficiency is given by the total number of events corresponding to 1 pe. The lower threshold for events counting as a 1 pe event is usually set in the valley between the first two peaks or at the $1 / 3$ pe level.

SiPMs: In the case of testing SiPMs, the QADC conversion rate is not able to keep up with the high signal rate. Therefore, the DAQ is substituted with a DRS4 evaluation board, manufactured by PSI (Paul Scherrer Institut). This board is essentially a fast and compact oscilloscope (0.7 GSPS to 5 GSPS) connected to a PC via USB. Since the intrinsic dark count rate of SiPMs is in the range of a few $\mathrm{MHz}$ at room temperature, and there is also a lot of optical crosstalk and thermal noise polluting the spectrum, a determination of the efficiency via the single photoelectron spectrum alone does not work [9]. For SiPMs the detection of photons follows Poisson statistics. From that the possibility to detect a photon can be expressed via the possibility to not detect any photon (pedestal) and the possibility to detect noise. A dark spectrum and a light spectrum (up to 4 pe to 10 pe level) have to be measured. Integrating the pedestals of both spectra gives the number of events in the pedestals ( $N_{\text {ped }}^{\text {dark }}$ and $N_{\text {ped }}$ ). The number of traces (dark spectrum) and respectively the trigger counter (light spectrum) provide the number of all events in each spectrum $\left(N_{\text {tot }}^{\text {dark }}\right.$ and $\left.N_{\mathrm{tot}}\right)$. The number of detected photons can be calculated [9]: $n_{\mathrm{pe}}=-\ln \left(\frac{N_{\mathrm{ped}}}{N_{\mathrm{tot}}}\right)+\ln \left(\frac{N_{\mathrm{ped}}^{\mathrm{dark}}}{N_{\mathrm{tot}}^{\mathrm{datk}}}\right)$. The 
efficiency of the SiPM is given by the ratio of the detected photons $n_{\mathrm{pe}}$ versus the number of single photons sent on the device.

Spectral light source For the purpose of a more versatile calibration set-up a new LED-array was designed, to have the possibility of quantum efficiency measurements with different wavelengths. The resulting light source is a spectral light source covering the spectral range of $310 \mathrm{~nm}$ to $425 \mathrm{~nm}$ with several LEDs (Figure 4 right).

\section{Comparison of MAPMT versus SiPM for EUSO-TA}

As already seen, the fluorescence spectrum has the main peaks in the range $310 \mathrm{~nm}$ to $430 \mathrm{~nm}$. MAPMTs and SiPMs have different wavelength sensitivity ranges. Indeed the sensitive range for MAPMTs is $280 \mathrm{~nm}$ to $650 \mathrm{~nm}$, while for SiPMs it is $320 \mathrm{~nm}$ to $900 \mathrm{~nm}$. In Figure 5 the overlay of the sensor efficiencies with the fluorescence spectrum ${ }^{2}$ versus wavelength are shown.
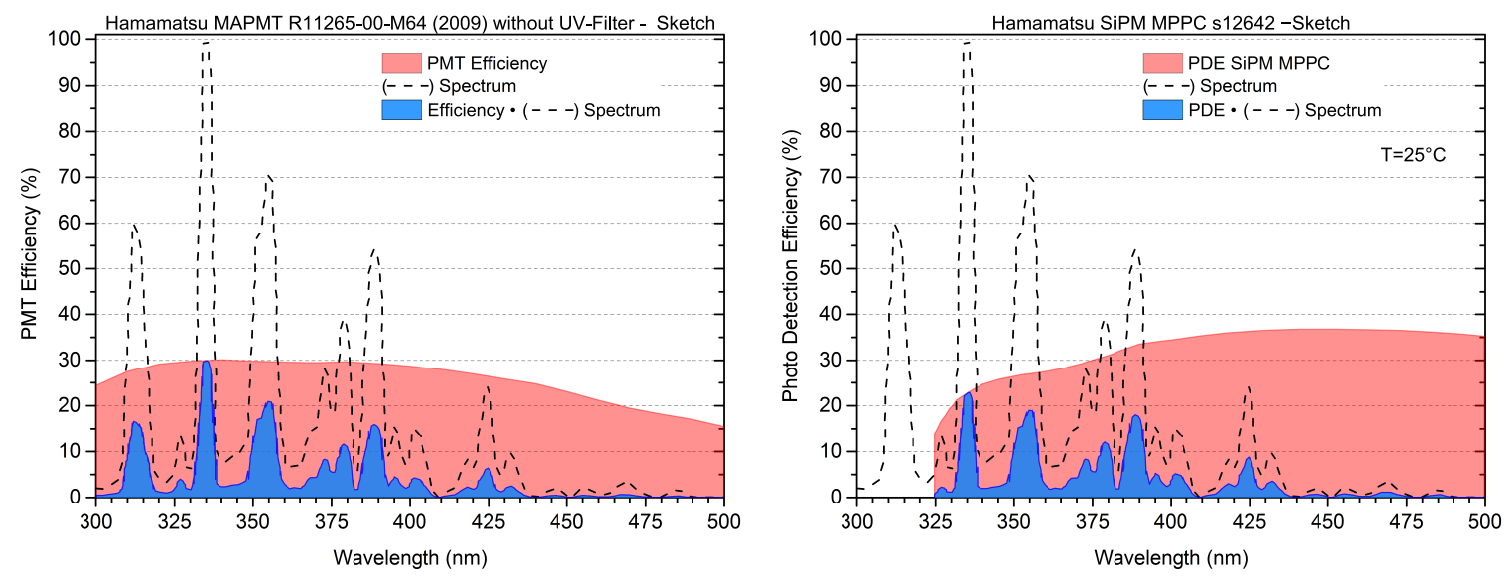

Figure 5: Left: Overlay of the MAPMT efficiency (Hamamatsu R11265-00-M64 prototype (2009)) with the nitrogen fluorescence spectrum versus wavelength. The blue area denotes the convolution of the N-spectrum with the MAPMT efficiency. It gives an estimation for the amount of signal that can be seen by the detector. Right: Same overlay for the SiPM efficiency (Hamamatsu MPPC s12642 (2014)). The first peak of the fluorescence spectrum can not be measured.

To test what the detector's response in front of an EAS would be, some simulations have been done considering SiPMs instead of MAPMTs. They are made using the analysis and simulation framework Offline, created for the Pierre Auger Observatory [10] and then adapted to the needs of the JEM-EUSO experiment [11].

In Figure 6 some preliminary results of the comparison between MAPMTs and SiPMs are shown. A vertical shower with energy $10^{20} \mathrm{eV}$ is simulated at about $20 \mathrm{~km}$ distance in front of EUSO-TA. The histogram on the left shows the number of photons arriving at the focal surface, to which the PDE of a MAPMT is applied, overlaid with the total number of photons at the focal surface (ratio of $28.88 \%$ ). The histogram on the right shows the same, but for the PDE of a SiPM (ratio of $24.67 \%$ ). In the case of the SiPM it is evident that the photons from the first peak of the

\footnotetext{
${ }^{2} \mathrm{http} / / /$ www.auger.org/technical_info/design_report.html - June 2015
} 
fluorescence spectrum are not detected. MAPMTs seem to be slightly better than SiPMs. However, if the sensitivity range of SiPMs is improved, they would be probably better than MAPMTs.
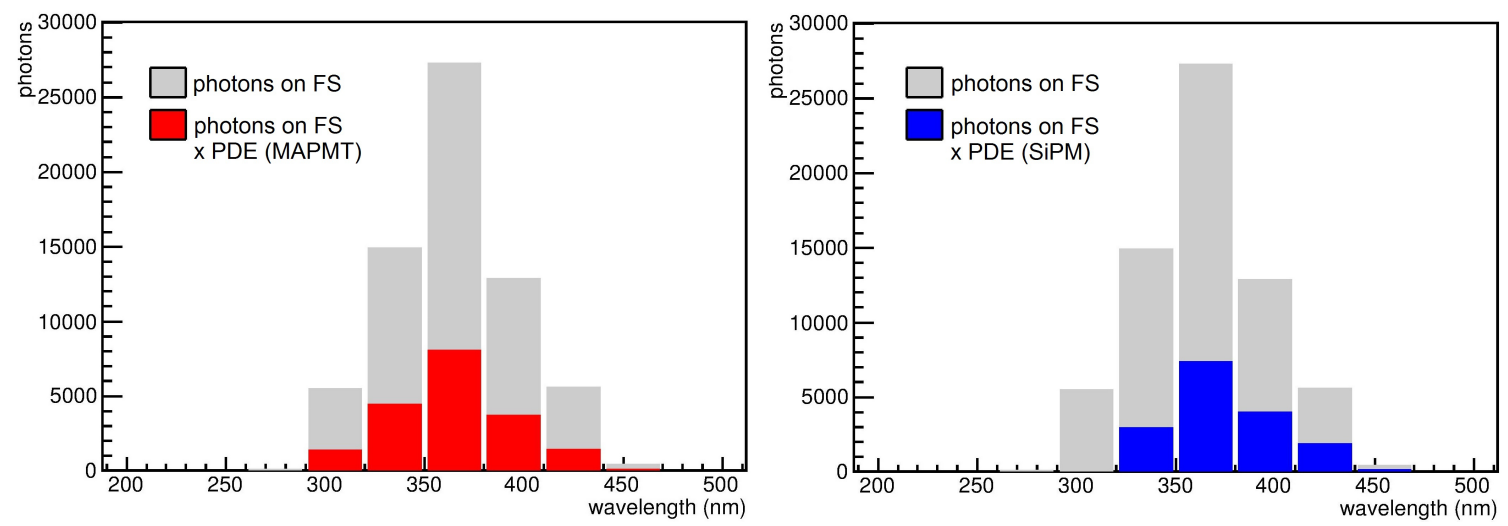

Figure 6: Left: Photons arriving at the focal surface as a function of their wavelength overlayed to the same photons to which the PDE (quantum efficiency multiplied by the collection efficiency, evaluated as $80 \%$ ) of a MAPMT (Hamamatsu R11265-00-M64) is applied. Right: The same, but applying the PDE of a SiPM (Hamamatsu S1036211100).

Acknowledgment: This work was partially supported by the 'Helmholtz Alliance for Astroparticle Physics HAP' funded by the Initiative and Networking Fund of the Helmholtz Association, Germany.

\section{References}

[1] J.H. Adams Jr. et al. - JEM-EUSO Collaboration, The JEM-EUSO Mission: Contributions to the ICRC 2013, proceedings of the ICRC2013 (2013) [astro-ph. IM/1307 . 7071].

[2] J.H. Adams Jr. et al. - JEM-EUSO Collaboration, Astroparticle Physics 44 (2013) 76 [astro-ph.HE/1305.2478].

[3] Y. Kawasaki and L.W. Piotrowski, Ground-based tests of JEM-EUSO components at the Telescope Array site, “EUSO-TA”, Experimental Astronomy (2015) 1.

[4] V.D. Kovaltchouk et al., Comparison of a silicon photomultiplier to a traditional vacuum photomultiplier, NIM A 538 (2005) 408.

[5] Hamamatsu Photonics K.K., TSV MPPC Array S12642-0808PB-50, Datasheet, 2014.

[6] C. Blaksley and P. Gorodetzky, A setup for the precision measurement of multianode photomultiplier efficiency, NIM A 764 (2014) 198 [physics.ins-det/1406.5800].

[7] C. Blaksley, Photodetection Aspects of JEM-EUSO and Studies of the Ultra-High Energy Cosmic Ray Sky, PhD thesis (2014) [astro-ph. IM/1406.5818].

[8] M. Ave et al. - AIRFLY Collaboration, Spectrally resolved pressure dependence measurements of air fluorescence emission with AIRFLY, NIM A 597 (2008) 41.

[9] P. Eckert et al., Characterisation studies of silicon photomultipliers, NIM A 620 (2010) 217 [physics.ins-det/1003.6071].

[10] J. Abraham et al., Properties and performance of the prototype instrument for the Pierre Auger Observatory, NIM A $\mathbf{5 2 3}$ (2004) 50.

[11] T.C. Paul - JEM-EUSO Collaboration, A new design for simulation and reconstruction software for the JEM-EUSO mission ID:0393, Proceedings of the ICRC2015 (2015). 